\title{
Correction to: Upscaling of LATP synthesis: Stoichiometric screening of phase purity and microstructure to ionic conductivity maps
}

\author{
Nikolas Schiffmann ${ }^{1}$ • Ethel C. Bucharsky ${ }^{1} \cdot$ Karl G. Schell $^{1} \cdot$ Charlotte A. Fritsch $^{2}$ - Michael Knapp ${ }^{2}$. \\ Michael J. Hoffmann ${ }^{1}$
}

Published online: 13 April 2021

(C) Springer-Verlag GmbH Germany, part of Springer Nature 2021

\section{Correction to: Ionics}

$$
\text { https://doi.org/10.1007/s11581-021-03961-x }
$$

The originally published version of this article contains an error in Eq. 2.

The correct Eq. 2 is shown below.

$\sigma=\frac{Z^{\prime}}{Z^{\prime 2}+Z^{\prime \prime 2}} * \frac{l}{A}$

The original article has been corrected.

Publisher's note Springer Nature remains neutral with regard to jurisdictional claims in published maps and institutional affiliations.

The online version of the original article can be found at https://doi.org/ 10.1007/s11581-021-03961-x

\footnotetext{
Nikolas Schiffmann

nikolas.schiffmann@kit.edu

1 Institute for Applied Materials - Ceramic Materials and

Technologies, Karlsruhe Institute of Technology (KIT),

Haid-und-Neu-Str. 7, 76131 Karlsruhe, Germany

2 Institute for Applied Materials - Energy Storage Systems, Karlsruhe Institute of Technology (KIT), Hermann-von-Helmholtz-Platz 1, 76344 Eggenstein-Leopoldshafen, Germany
} 